\title{
Directional Channel Characteristics from Microcell Measurement and Simulation
}

\author{
Navarat Lertsirisopon, Gilbert Siy Ching, \\ Mir Ghoraishi, Jun-ichi Takada \\ Department of International Development Engineering, \\ Tokyo Institute of Technology, Tokyo, Japan \\ Emails: \{joy,gilbert,mir,takada\}@ap.ide.titech.ac.jp
}

\author{
Ichiro Ida \\ Yasuyuki Oishi \\ Fujitsu Ltd. \\ Email:ida.ichirou@jp.fujitsu.com \\ Email:yasu@labs.fujitsu.com
}

\begin{abstract}
In this paper, the channel predicted by using a raytracing simulator is presented and compared to microcellular measurement results performed inside a university campus. For data processing, the conventional beamforming is utilized to compare the directional wideband channel properties of both simulation and measurement results. Ray-tracing algorithm can investigate most of the radio propagation mechanisms. However, there are some scattering effects that are not included. Therefore, the objective of this study is to analyze and compare directional wideband channel characteristics such as azimuth-delay spectrum and azimuth-coelevation spectrum from the ray-tracing results with measurements to gain insights on the significant propagation mechanisms in microcell scenarios.
\end{abstract}

\section{INTRODUCTION}

In mobile communication systems, multiple input multiple output (MIMO) systems employing antenna arrays at both the mobile station (MS) and base station (BS) have recently emerged as a key technology to address the increasing demands for high data rate applications as well as the reliability of the systems [1] - [2]. The performance of MIMO systems depends much on the directional and delay characteristics of the channel, and models of wireless propagation channel should include the directions of arrival (DoA), directions of departure (DoD) and time delay of arrival (TDoA) of the multipath components. Therefore, to reflect the doubledirectional wideband structure of the radio channel in a considered propagation environment, realistic channel models are required instead of the conventional channel models [3].

Ray-tracing algorithms are popular approaches for propagation prediction and modeling since it can investigate most of the radio propagation mechanisms of wireless channels. It is a common knowledge that ray-tracing algorithms [4]-[5] can predict the elementary propagation mechanisms such as specular reflection from wall surfaces and diffraction from building edges. In microcell scenarios, however, there are some scattering effects [6]-[8] that are not yet included in the algorithm. Therefore, the objective of this study is to analyze and compare directional wideband channel characteristics from the ray-tracing results with measurements to gain insights on the significant propagation mechanisms in microcell scenarios.

\section{Measurement SCEnARio And EQuipment}

The microcell measurement was carried out in O-okayama campus of Tokyo Institute of Technology [9]. In the following

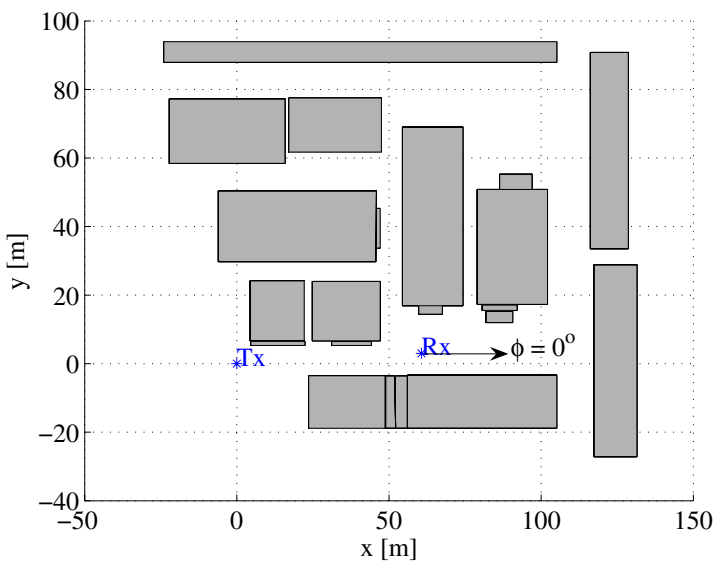

Fig. 1. Measurement scenario.

subsections we will describe the measurement scenario and employed equipment.

\section{A. Scenario}

In this measurement, the Tx antenna corresponds to the base station (BS) while the Rx antenna corresponds to the mobile station (MS). The BS antenna was fixed and mounted on a tripod with a height of $1.79 \mathrm{~m}$ from the ground while the MS antenna with LOS to the BS, was mounted on a cart with a height of $1.65 \mathrm{~m}$. The 0 degree of the MS array antenna is pointing in the same direction as the $\mathrm{x}$-axis as illustrated in Fig. 1. The distance between BS and MS was $60 \mathrm{~m}$.

\section{B. Channel Sounding}

The Medav RUSK Fujitsu channel sounder [10] was employed to accomplish the measurements and the related parameters can be found in Table I. A periodic multicarrier frequency signal was utilized as the test signal at the center frequency of $4.5 \mathrm{GHz}$ allowing real time measurements of the channel impulse response with a $120 \mathrm{MHz}$ bandwidth resulting in a propagation delay resolution of $8.3 \mathrm{~ns}$. The BS antenna is a $2 \times 4$ uniform rectangular antenna array of dual-polarized patch antenna elements whereas the MS antenna array is a $2 \times 24$ stacked uniform circular antenna array with dual-polarized patches constituting 96 elements in total. Reference clocks at both BS and MS ensure timing and 
TABLE I

SPECIFICATIONS OF EXPERIMENT

\begin{tabular}{ll} 
Center frequency & $4.5 \mathrm{GHz}$ \\
Bandwidth & $120 \mathrm{MHz}$ \\
Delay resolution & $8.3 \mathrm{~ns}$ \\
Excess delay & $3.2 \mu \mathrm{s}$ \\
Tx power & $40 \mathrm{dBm}$ \\
Tx antenna & rectangular array, $2 \times 4$ dual polarized \\
Tx antenna height & $1.79 \mathrm{~m}$ \\
Rx antenna & cylindrical array, $2 \times 24$ dual polarized \\
Rx antenna height & $1.65 \mathrm{~m}$ \\
\hline \hline
\end{tabular}

switching frame synchronization for the whole measurement durations. Fast switches were used in both Tx and Rx array antennas to switch the antenna ports to sample the received signal in the spatial domain. Accordingly, the total number of channels is 1536 including all combination of vertical and horizontal polarizations.

\section{PATh DEtermination USING RAY-TRACING SIMULATOR}

In this paper, the ray-tracing simulator called "Raplab" [11] is used to predict detailed path parameters, i.e. complex path gain, DoD and DoA in azimuth and elevation, and TDoA. The ray-tracing simulator is able to identify rays with multiple reflections, multiple diffractions and combinations of both. To trace rays from a source to the observation point, the image method is utilized to find the ray paths and the angle based on Snell's law of reflection. For example, given a source point and a wall, the reflected ray from the wall can be considered as the ray radiated from a virtual source point, called the image source. For the calculation of electromagnetic wave theory, the formulation of reflected and diffracted rays are carried out based on geometrical optics (GO), and the uniform theory of diffraction (UTD) [12].

The ray-tracing scenario is modeled the same as the measurement scenario (Fig. 1). Buildings are modeled as rectangular boxes by assuming the material parameters of concrete $\left(\epsilon_{r}=6.765, \mu_{r}=1, \sigma=0.0023[\mathrm{~s} / \mathrm{m}]\right.$, thickness $=0.1$ $[\mathrm{m}])$. The ideal isotropic antennas are assumed to simulate an antenna independent channel. By tilting the BS and MS antenna, both vertical and horizontal polarizations can be obtained. The maximum reflection order of a path is set to 2 and the maximum diffraction order to 1 . In each MS position, Raplab generates a list of all propagation paths and its path parameters.

\section{Channel Data Processing Reconstruction}

Although the authors can compare the ray tracing result directly with estimated ray parameters from the measurements results by maximum likelihood estimation [13], this comparison is not effective due to the different resolution limit for these two approaches.The angular and delay resolution of the
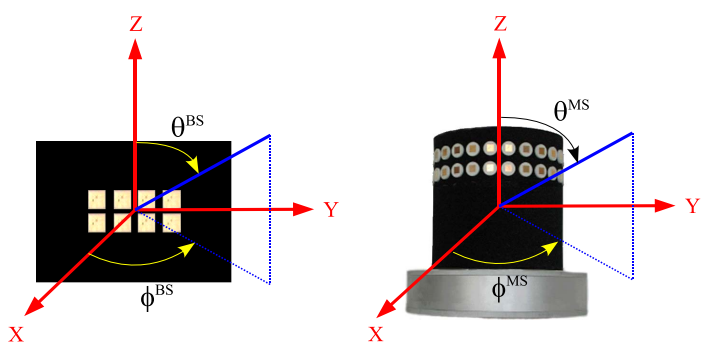

Fig. 2. Coordinate system at BS and MS, respectively

former can be infinitesimally small, but that of the latter is physically limited by the bandwidth and the size of the array antennas. Therefore, the authors are going to consider the limitation of the latter, and the beamforming/matched filtering output of the channel sounder and its simulation are used for the comparison.

To simulate the measurement by using ray tracing output, the array frequency response $\mathbf{h}_{\mathrm{RT}}$ is constructed as

$$
\begin{aligned}
\mathbf{h}_{\mathrm{RT}} & =\sum_{l=1}^{L} \gamma_{\mathrm{VV}, l} \cdot\left(\mathbf{h}_{\mathrm{F}, l} \otimes\left(\mathbf{h}_{\mathrm{BS}, \mathrm{V}, l} \otimes \mathbf{h}_{\mathrm{MS}, \mathrm{V}, l}\right)\right) \\
& +\gamma_{\mathrm{HV}, l} \cdot\left(\mathbf{h}_{\mathrm{F}, l} \otimes\left(\mathbf{h}_{\mathrm{BS}, \mathrm{V}, l} \otimes \mathbf{h}_{\mathrm{MS}, \mathrm{H}, l}\right)\right) \\
& +\gamma_{\mathrm{VH}, l} \cdot\left(\mathbf{h}_{\mathrm{F}, l} \otimes\left(\mathbf{h}_{\mathrm{BS}, \mathrm{H}, l} \otimes \mathbf{h}_{\mathrm{MS}, \mathrm{V}, l}\right)\right) \\
& +\gamma_{\mathrm{HH}, l} \cdot\left(\mathbf{h}_{\mathrm{F}, l} \otimes\left(\mathbf{h}_{\mathrm{BS}, \mathrm{H}, l} \otimes \mathbf{h}_{\mathrm{MS}, \mathrm{H}, l}\right)\right)
\end{aligned}
$$

where $L$ is the number of paths, the first and the second subscripts of polarimetric complex path weights $(\gamma)$ indicate the MS and BS, respectively. $\gamma_{\mathrm{HH}, l}$ and $\gamma_{\mathrm{VV}, l}$ are the copolarization components of horizontal polarization(HP) and vertical polarization(VP) transmission, respectively, while, $\gamma_{\mathrm{HV}, l}$ and $\gamma_{\mathrm{VH}, l}$ are those of cross polarization components. Operator $\otimes$ means the Kronecker product. $\mathbf{h}_{\mathrm{F}, l}, \mathbf{h}_{\mathrm{BS}, \alpha, l}$, and $\mathbf{h}_{\mathrm{MS}, \alpha, l}(\alpha=\mathrm{V}, \mathrm{H})$ are frequency, BS and MS array transfer function of $l$ th path defined as

$$
\begin{aligned}
\mathbf{h}_{\mathrm{F}, l} & =\mathbf{h}_{\mathrm{F}}\left(\tau_{l}\right) \\
\mathbf{h}_{\mathrm{BS}, \alpha, l} & =\mathbf{h}_{\mathrm{BS}, \alpha}\left(\theta_{l}^{\mathrm{BS}}, \phi_{l}^{\mathrm{BS}}\right) \\
\mathbf{h}_{\mathrm{MS}, \alpha, l} & =\mathbf{h}_{\mathrm{MS}, \alpha}\left(\theta_{l}^{\mathrm{MS}}, \phi_{l}^{\mathrm{MS}}\right)
\end{aligned}
$$

where

$$
\begin{aligned}
\mathbf{h}_{\mathrm{F}}\left(\tau_{l}\right) & =e^{j 2 \pi \cdot \tau_{l} \cdot\left(f_{0}+\Delta f \cdot \mathbf{m}_{f}\right)} \\
\mathbf{m}_{f} & =\left[-\frac{M_{f}-1}{2}, \ldots, \frac{M_{f}-1}{2}\right]^{T} \\
B W & =\left(M_{f}-1\right) \cdot \Delta f
\end{aligned}
$$

$\mathbf{h}_{\mathrm{BS}, \alpha}\left(\theta_{l}^{\mathrm{BS}}, \phi_{l}^{\mathrm{BS}}\right)$ and $\mathbf{h}_{\mathrm{MS}, \alpha}\left(\theta_{l}^{\mathrm{MS}}, \phi_{l}^{\mathrm{MS}}\right)$ are known as the array calibration data. The definitions of $\theta$ and $\phi$ components for BS and MS are illustrated in Figs. 2. Since the measured and simulated data are available, the beamforming in the angular domain and the matched filtering in the delay domain are conducted by using the weight vector $\mathbf{w}(\mathbf{s}) ; \mathbf{s}=$ $\left[\tau, \theta^{\mathrm{BS}}, \phi^{\mathrm{BS}}, \alpha^{\mathrm{BS}}, \theta^{\mathrm{MS}}, \phi^{\mathrm{MS}}, \alpha^{\mathrm{MS}}\right]$ as

$$
\begin{aligned}
\mathbf{w}(\mathbf{s}) & =\mathbf{h}_{\mathrm{F}}(\tau) \otimes \mathbf{h}_{\mathrm{BS}, \alpha^{\mathrm{BS}}}\left(\theta^{\mathrm{BS}}, \phi^{\mathrm{BS}}\right) \\
& \otimes \mathbf{h}_{\mathrm{MS}, \alpha^{\mathrm{MS}}}\left(\theta^{\mathrm{MS}}, \phi^{\mathrm{MS}}\right)
\end{aligned}
$$




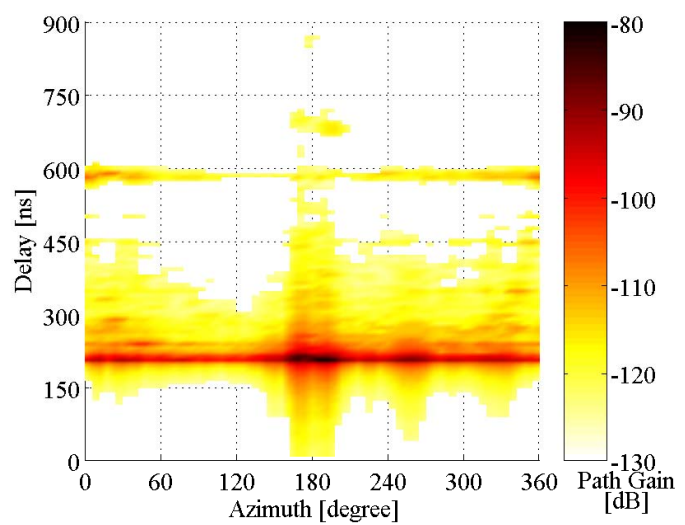

Fig. 3. Azimuth Delay Spectrum: Experiment

and the resultant spectrum $P(\mathbf{s})$ is given as

$$
P(\mathbf{s})=\frac{\left|\mathbf{w}^{H}(\mathbf{s}) \mathbf{h}_{\mathrm{RT}}\right|^{2}}{\mathbf{w}^{H}(\mathbf{s}) \mathbf{w}(\mathbf{s})}
$$

Beamforming is applied in every $6^{\circ}$ for the whole azimuth range (from $0^{\circ}$ to $360^{\circ}$ ), however, for the coelevation, beamforming is applied in the range from $30^{\circ}$ to $150^{\circ}$ according to antenna limitations. The comparison between experimental and simulation results are discussed in the next section.

\section{RESULTS AND DISCUSSION}

\section{A. Azimuth-Delay Spectrum}

The experimental and the simulation results of the azimuthdelay spectrum defined as Eq.(4) are shown in Figs. 3 and 4, respectively.

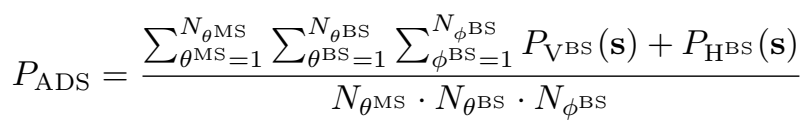

By comparing both results, the ray-tracing simulation can be seen to predict the arrival of strong signals around $180^{\circ}$. A strong delayed path near $0^{\circ}$ is also predicted corresponding to the building in front of MS. In the next subsections, the azimuth spectrum and delay spectrum of both cases are compared separately.

a) Azimuth Spectrum: Azimuth spectrum obtained by summing up the power of azimuth-delay spectrum with respect to the delay time is defined as

$$
P_{\mathrm{AS}}\left(\phi^{\mathrm{MS}}\right)=\sum_{\tau=1}^{N_{\tau}} P_{\mathrm{ADS}}\left(\phi^{\mathrm{MS}}, \tau\right)
$$

Figure 5 shows the azimuth profiles obtained from the experiment as the solid line and from the simulation as the dotted line. From Fig. 5, the arrival waves within the range from $138^{\circ}$ to $216^{\circ}$ are in agreement with respect to the shape of the spectrum. At $0^{\circ}$ some power is simulated by the ray tracing algorithm corresponding to the building in front of MS. The remaining angles which do not match corresponds to the right and left side of the MS.

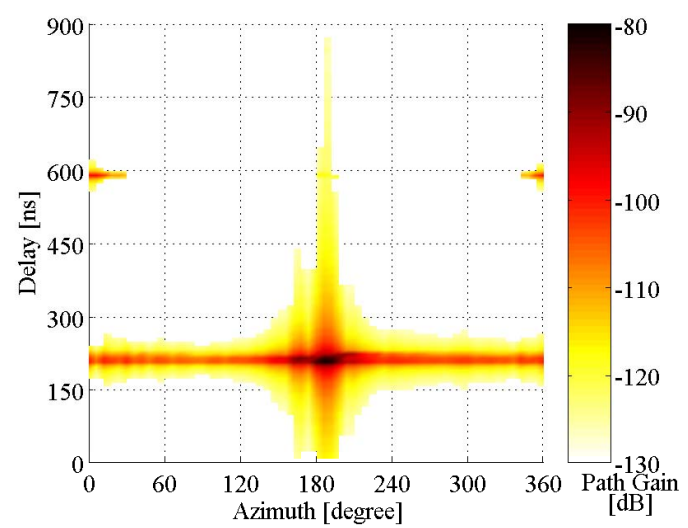

Fig. 4. Azimuth Delay Spectrum: Simulation

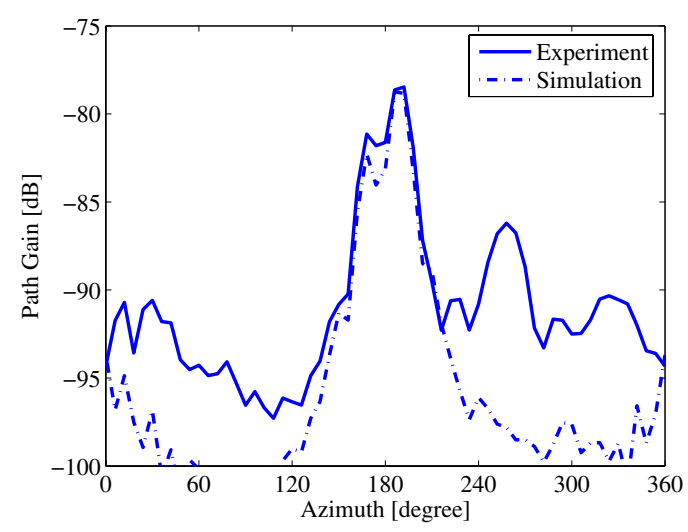

Fig. 5. Azimuth Spectrum

b) Delay Spectrum: Delay spectrum obtained by summing up the azimuth-delay spectrum with respect to the azimuth angle is defined as

$$
P_{\mathrm{DS}}(\tau)=\sum_{\phi^{\mathrm{MS}}=1}^{N_{\phi^{\mathrm{MS}}}} P_{\mathrm{ADS}}\left(\phi^{\mathrm{MS}}, \tau\right)
$$

Figure 6 shows the delay spectrum obtained from the experiment as the solid line and from the simulation as the dotted line. From Fig. 6, the ray-tracing simulation can predict accurately the two major peaks around 200 and $600 \mathrm{~ns}$ in the delay spectrum. For the $200 \mathrm{~ns}$ peak, it is composed of the LOS path and other paths close to it like reflections from the building walls in between BS and MS. The $600 \mathrm{~ns}$ peak corresponds to the previously mentioned building in front of MS. However, other minor peaks which might correspond also to the right and left side of MS, cannot be predicted.

\section{B. Azimuth-Coelevation Spectrum}

In order to have more idea on the possible propagation mechanisms, the azimuth-coelevation spectrum from both experimental and simulation results are shown in Figs. 7 and 8, respectively by specifying the delay domain at the maximum 


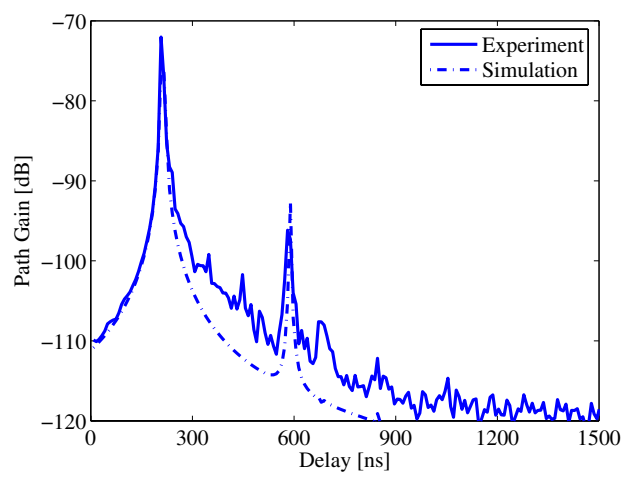

Fig. 6. Delay Spectrum

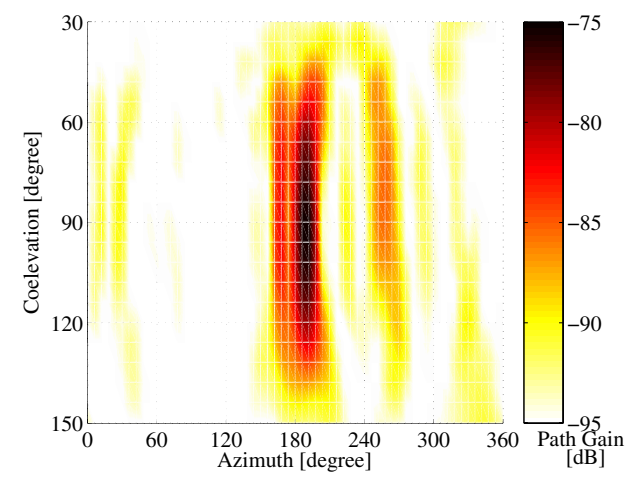

Fig. 7. Azimuth Coelevation Spectrum at LOS delay: Experiment

path gain occurring at $207 \mathrm{~ns}$ which can be defined as

$P_{\mathrm{ACS}}\left(\tau_{l @ \mathrm{LOS}}\right)=\frac{\sum_{\theta^{\mathrm{BS}}=1}^{N_{\mathrm{BS}^{\mathrm{BS}}}} \sum_{\phi^{\mathrm{BS}}=1}^{N_{\phi^{\mathrm{BS}}}} P_{\mathrm{VBS}}\left(\mathbf{s}, \tau_{l}\right)+P_{\mathrm{H}^{\mathrm{BS}}}\left(\mathbf{s}, \tau_{l}\right)}{N_{\theta^{\mathrm{BS}}} \cdot N_{\phi^{\mathrm{BS}}}}$

By comparing both results, the ray-tracing simulation can be seen to precisely estimate the coelevation of arrival waves within $50^{\circ}$ to $130^{\circ}$ in the azimuth range of the strong arrival waves. However, the ray tracing simulation cannot predict the other regions since the minor peaks cannot be detected as seen from the azimuth spectrum results (Fig. 5).

\section{CONCLUSION}

In this paper, a ray tracing simulator was used to predict the path parameters of a LOS microcellular environment inside a university campus, and the results were applied to reconstruct the channel impulse response. For data processing, the conventional beamforming is utilized to extract the directional wideband channel properties. These are then compared to measured results. The results have shown that the directional wideband channel properties extracted from the ray-tracing result can predict the major peaks with those from the experiment. In the future, further investigations will be made to find out the significant propagation mechanisms that cause the other peaks not to be predicted.

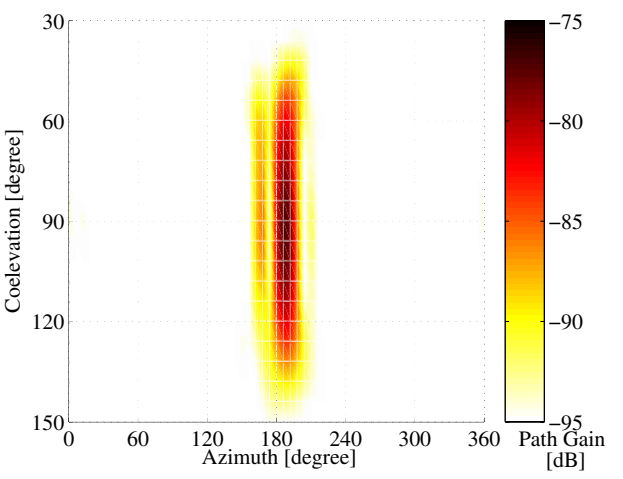

Fig. 8. Azimuth Coelevation Spectrum at LOS delay: Simulation

\section{ACKNOWLEDGMENT}

This research is supported by the National Institute of Information and Communications Technology of Japan (NICT).

\section{REFERENCES}

[1] 3GPP, TR 25.996 "Spatial channel model for MIMO simulations," V6.1.0, Sep 2003.

[2] A. F. Molisch, "A generic model for MIMO wireless propagation channels in macrocells and microcells," IEEE Transaction on Signal Processing, Jan 2004.

[3] H. Asplund, A. Molisch, M. Steinbauer, and N. Mehta, "Clustering of scatterers in mobile radio channels - evaluation and modeling in the COST259 directional channel model," in Proc. IEEE International Conference Communication (ICC 2002), Apr. 2002, vol. 2, pp. 901-905.

[4] H. Zhu, J. Takada, K. Araki, and T. Kobayashi, "A ray-tracing-based characterization and verification of the spatio-temporal channel model for future wideband wireless systems," IEICE Transactions on Communications, vol. E84-B, no. 3, pp. 644-652, Mar. 2001.

[5] J. Takada, J. Fu, H. Zhu, and T. Kobayashi, "Spatio-temporal channel characterization in a suburban non line-of-sight microcellular environment,' IEEE Journal on Selected Areas in Communications, vol. 20, no. 3, pp. 532-578, Apr. 2002.

[6] H. Li, C. Chen, T. Liu, and H. Lin, "Applicability of ray-tracing technique for the prediction of outdoor channel characteristics," IEEE Transactions on Vehicular Technology, vol. 49, no. 6, pp. 2336-2349, Nov. 2000.

[7] H. Budiarto, K. Horihata, K. Haneda, and J. Takada, "Experimental study of non-specular wave scattering from building surface roughness for the mobile propagation modeling," IEICE Transactions on Communications, vol. E87-B, no. 4, pp. 958-966, Apr. 2004.

[8] M. Ghoraishi, J. Takada, and T. Imai, "Identification of scattering objects in the microcell urban mobile propagation channel," IEEE Transactions on Antennas and Propagation, Nov. 2006.

[9] N. Lertsirisopon, G. S. Ching, M. Ghoraishi, J. Takada, I. Ida, and Y. Oishi, "Double directional channel characteristics of microcell environment inside university campus," IEICE Technical Report, AP2006-139, Feb. 2007.

[10] M. Landmann, K. Sivasondhivat, J. Takada, I. Ida, and R. S. Thomä, "Polarisation behaviour of discrete multipath and diffuse scattering in urban environments at $4.5 \mathrm{GHz}$," EURASIP Journal on Wireless Communications and Networking, vol. 2007, Article ID 57980.

[11] Radio Propagation Laboratory - Raplab -, Available: http://www4.kke.co.jp/raplab/

[12] R. J. Luebbers, "Finite conductivity uniform GTD versus knife-edge diffraction in prediction of propagation path loss," IEEE Transactions on Antennas and Propagation, vol. AP-32, pp. 70-76, Jan. 1984.

[13] R. S. Thomä, D. Hampicke, M. Landmann, A. Richter, and G. Sommerkorn, "Measurement-based parametric channel modeling (MBPCM)," in ICEAA, Sep. 2003. 